\title{
MEDICIÓN DEL TIPO DE CAMBIO REAL: UNA COMPARACIÓN DE LAS MEDIDAS OFICIALES EN MÉXICO
}

\section{MEASURING THE REAL EXCHANGE RATE: A COMPARISON OF OFFICIAL MEASURES IN MEXICO}

\section{RESUMEN}

Este trabajo compara diferentes medidas del tipo de cambio real (TCR) para México. La primera parte presenta una descripción de cinco diferentes medidas del TCR, con énfasis en las ventajas y desventajas teóricas de cada una de ellas. La segunda parte presenta una comparación econométrica de las alternativas analizadas con el objetivo de tener un soporte empírico que nos pueda decir cuál de estos índices puede ser preferido en relación con los otros. El análisis se realizó bajo el criterio de que una buena medida del TCR debe ser sensible a cambios en la oferta de exportaciones, demanda de importaciones y de los flujos de capital.

Palabras clave: tipo de cambio real, series de tiempo, FMI, OCDE, Banco de México, SDRWPI.

Clasificación JEL: F31, C22, E02

\footnotetext{
El autor agradece a la Dra. Sylvia B. Guillermo Peón de la Benemérita Universidad Autónoma de Puebla por su invaluable asesoría durante el desarrollo del presente trabajo.
} 


\begin{abstract}
This paper compares different measures of the real exchange rate (RER) for Mexico. The first part of the paper presents an overview of five different measures of RER, emphasizing the theoretical advantages and disadvantages of each. The second part presents an econometric comparison of the alternatives analyzed in order to have empirical support that can tell us which of these indices may be preferred on the other. The analysis is made under the criterion that a good measure of RER should be sensitive to changes in export supply, import demand and capital flows.
\end{abstract}

Keywords: real exchange rate, time series, Bank of Mexico, SDRWPI. 


\section{INTRODUCCIÓN}

El tipo de cambio real (TCR) es una de las variables más importantes para cualquier economía y se ha convertido en tema central de las discusiones sobre política económica, tanto en los países desarrollados como en los que están en vías de serlo. Su importancia radica en el hecho de que es el precio real que hace que la balanza de pagos esté en equilibrio, es decir, es el precio real que hace que la oferta y la demanda reales de divisas se encuentren en equilibrio. Por lo anterior, es necesario tener una adecuada medida que sea capaz de captar de la mejor manera posible el efecto de los cambios en las variables que consideramos fundamentos del TCR.

Para México existen diversas medidas del tipo de cambio real que son publicadas por diferentes instituciones oficiales como: Banco de México, Fondo Monetario Internacional y la Organización para la Cooperación y el Desarrollo Económico. ${ }^{1}$

En este trabajo se presentan las metodologías utilizadas por los organismos arriba mencionados para la estimación de los tipos de cambio real, junto con una quinta metodología propuesta por Harberger (1988). El objetivo es poderlas comparar para saber cuál de ellas es la más apropiada para medir el TCR en México. Para ello estimamos que, una buena medida del tipo de cambio real, debe ser sensible a los cambios en las variables que consideramos sus fundamentos. Es decir, una buena medida del TCR debe ser capaz de captar los cambios en la oferta real de exportaciones, en la demanda real de importaciones y en los flujos de capital. Al tener este como objetivo las técnicas econométricas serán la herramienta que proporcione elementos en favor de una u otra medida alternativa del TCR. Relacionamos el TCR con las variables explicativas de la siguiente manera:

$$
T C R_{t}=\operatorname{TCR}\left(X G_{\mathrm{t}-1}, M G_{t}, X M G_{t}\right)
$$

Dónde: $\mathrm{TCR}_{\mathrm{t}}$ es el logaritmo del índice de TCR en el tiempo $t ; \mathrm{XG}_{\mathrm{t}-1}$ es la tasa de exportaciones (de bienes y servicios) respecto del PIB en el

${ }^{1}$ Para México la OCDE publica dos índices de tipo de cambio real. 
periodo $t-1 ; \mathrm{MG}_{\mathrm{t}}$ es la tasa de importaciones (de bienes y servicios) respecto del PIB el tiempo $t$; y $\mathrm{XMG}_{\mathrm{t}}$ es la tasa de flujos de capital respecto del PIB (definido como la diferencia entre las exportaciones e importaciones) en el tiempo $t$.

El objetivo del análisis econométrico —una vez definida la relación adecuada entre las variables - será el de saber que tan bien los cambios en el TCR son explicados por cambios en las variables consideradas como sus fundamentos. Para ello, la medida de bondad de ajuste $R^{2}$ y la significancia de los coeficientes serán la clave para nuestra comparación.

El trabajo está estructurado de la siguiente manera: la sección 2 presenta las diferentes metodologías de medición del TCR, la 3 contempla la metodología utilizada para la comparación de los índices alternativos del TCR, para finalizar la sección 4 contiene las principales conclusiones.

\section{MEDIDAS DE TIPO DE CAMBIO REAL PARA MÉXICO}

\subsection{Tipo de cambio real calculado por el Banco de México (TCR-BX)}

El Banco de México calcula y publica mensualmente su índice de tipo de cambio real, ${ }^{2}$ este índice es definido como el cociente del índice mundial de precios al consumidor en pesos de 111 países, dividido por el índice nacional de precios al consumidor. ${ }^{3}$ El índice mundial de precios al consumidor se construyó a partir de un agregado geométrico de los índices de precios correspondientes a los 111 países, para ello se utilizó como ponderador el PIB nominal de 1990 de cada país, como porcentaje del PIB mundial, expresado en dólares estadounidenses.

De este modo, el índice de tipo de cambio real empleado por el Banco de México queda expresado de la siguiente manera:

$$
T C R-B X=\frac{P^{*}}{P_{I N P C}} * \frac{E}{E^{*}}
$$

\footnotetext{
${ }^{2}$ Véase www.banxico.org.

${ }^{3}$ Inicialmente fueron 41 países, posteriormente se ajustó su número hasta que el Banco de México optó por el índice de tipo de cambio real con 111 países. La lista de países incluidos en este índice se encuentra disponible en la página web del Banco de México.
} 
Donde $P^{*}$ es el índice de precios al consumidor de la cesta de bienes de los países, el cual constituye un agregado geométrico; $P_{I N P C}$ es el índice nacional de precios al consumidor, INPC, en México; $E$ es el índice de tipo de cambio nominal de pesos por dólar americano y es el índice de tipo de cambio nominal del conjunto de países en monedas por dólar americano, también integrado como un agregado geométrico.

De acuerdo con Carballo y Uzúa (2008), esta metodología es anómala por dos razones: a) en lugar de ponderar cada moneda mediante la participación del país respectivo en los flujos comerciales de México, se emplea su participación en el PIB mundial y b) se utilizan los índices de precios de un sinnúmero de países, muchos de los cuales comercian poco o nada con México.

Una tercera crítica, considero, es el hecho de que el año utilizado para el cálculo de la ponderación del PIB de los países que integran el índice sea 1990, cuando la realidad muestra que la participación del PIB de los países respecto del PIB mundial ha cambiado significativamente durante los últimos 20 años. Así, por ejemplo, la participación del PIB de Estados Unidos en el mundo en 1990 era de 26.26 y para 2010 fue de $23.13 \%$; Alemania, Reino Unido, Japón y Francia aportaban 7.82, 4.62, 13.96 y 5.68 por ciento del PIB mundial en 1990, para 2010 cambiaron a 5.24, 3.56, 8.72 y 4.06, respectivamente. El caso más significativo es el de China, cuya participación en el PIB mundial en 1990 fue de $1.63 \%$ y para 2010 se elevó a 9.32 por ciento. ${ }^{4}$ Lo anterior debe considerarse una deficiencia importante en el indicador de TCR calculado por el Banco de México, ya que la participación del PIB, respecto del PIB mundial, de cada país que integra el índice, y que se utiliza para su cálculo, ya es obsoleta y podría no estar reflejando las ponderaciones de cada país de una forma adecuada en la estimación del TCR. Esto estaría llevándonos a evaluaciones incorrectas sobre el desarrollo de dicho indicador.

\footnotetext{
${ }^{4}$ Fuente: Estadísticas del Banco Mundial. Varios años.
} 


\subsection{Tipo de cambio real basado en los índices relativos de precios al consumidor publicado por el FMI (TCR-FMI)}

El índice de tipo de cambio real basado en los índices relativos de precios al consumidor es el índice que el FMI publica periódicamente en sus Estadísticas financieras internacionales (IFS, por sus siglas en inglés) para 164 de sus países miembros (entre ellos México), utilizando datos sobre consumo y comercio de manufacturas de 1999 a 2001. Este indicador toma en cuenta la competencia entre importaciones y sustitutos de importaciones producidos localmente, la competencia entre las exportaciones propias y los bienes externos producidos localmente y la competencia entre las exportaciones propias frente a las exportaciones de otros países en "terceros mercados".

Dado un conjunto de ponderaciones para el país $i$ sobre sus socios comerciales $\left(W_{i j}\right.$ para $\left.j \neq i\right)$, el TCR-FMI es calculado como un promedio geométrico de los tipos de cambio real bilaterales entre el país local y sus socios comerciales. Específicamente, el TCR-CPI ${ }_{\mathrm{FMI}}$ para el país $i$ es calculado de la siguiente manera:

$$
T C R-F M I_{i}=\Pi_{j \neq i}\left[\frac{P_{i} R_{i}}{P_{j} R_{j}}\right]^{W_{i j}}
$$

donde $j$ es un índice que corre sobre los socios comerciales del país $i ; W_{i j}$ es la ponderación de competitividad que el país $i$ le asigna al país $j$; $P_{i}, P_{j}$ son los índices de precios al consumidor para el país $i$ y $j$ respectivamente. Finalmente, $R i, R j$ son los tipos de cambio nominales bilaterales del país $i$ y $j$ medidos en dólares por moneda local.

El esquema de ponderación $W_{i j}$ es muy importante en esta metodología ya que determina cómo las variaciones en el tipo de cambio nominal y en los precios al consumidor en diferentes países externos tienen un impacto sobre la posición competitiva del país local. Por esto, el FMI calcula para cada país ponderaciones de competitividad (normalizadas) de los países socios en tres categorías: materias primas (commodities), manufacturas y servicios comerciales no turísticos. ${ }^{5}$ Los tres conjuntos de

\footnotetext{
${ }^{5}$ Para 48 de sus países miembros el FMI agrega una cuarta categoría que es la de servicios turísticos, el esquema de ponderación para ésta es similar al de las manufacturas, y se ha excluido
} 
ponderaciones son agregados para obtener un total del conjunto de ponderaciones de competitividad — de nuevo para cada país - , cada una de estas ponderaciones en lo individual es multiplicada por la proporción del total del comercio que le corresponde a cada una de las tres categorías.

Si expresamos lo anterior algebraicamente tendremos:

$$
W_{i j}=\left(\propto_{M}+\propto_{S}\right) w_{i j}(M)+\propto_{C} w_{i j}(C)
$$

dónde, $w_{i j}(M), w_{i j}(C)$ son las ponderaciones basadas en el comercio de manufacturas y materias primas, respectivamente, que el país $i$ asigna al país $j, \alpha_{M}, \alpha_{S}$ y $\alpha_{C}$ son los porcentajes del comercio en manufacturas, servicios comerciales no turísticos y materias primas, del comercio exterior del país $i$, donde el comercio exterior es la suma de las tres categorías.

Esta medida del TCR presenta limitaciones tanto metodológicas como teóricas. En relación con las primeras, Zanello y Desruelle (1997) comentan que son al menos dos asuntos básicos metodológicos que surgen en el cálculo del TCR-FMI:

1. La determinación de una significativa estructura de ponderación de competitividad $\mathrm{y}$,

2. La elección de un nivel de desagregación en la definición de productos y mercados.

En lo que se refiere a las limitaciones teóricas, Guillermo (2000) menciona que al menos son dos las que este índice presenta.

a) El uso de indicadores de competitividad expresados como un índice en un año base debe manejarse con cautela cuando se trata de hacer comparaciones entre países. Si el índice tiene un año base, la diferencia relativa en competitividad entre países no se observa en el año base de elección y

aquí porque México no se encuentra dentro de los 48 países. La ecuación 2 con esta cuarta categoría quedaría de la siguiente manera: $W_{i j}=\left(\propto_{M}+\propto_{S}\right) w_{i j}(M)+\propto_{C} w_{i j}(C)+\propto_{T} w_{i j}(T)$. Para mayores detalles de cómo se calcula la ponderación de competitividad para servicios turísticos puede verse Bayoumi, Lee y Jayanthi (2005). 
b) La interpretación de este índice como tipo de cambio real es complicada porque, al parecer, el TCR-CPI pone más énfasis en ser un indicador de competitividad y no el precio real que equilibre la balanza de pagos, que es el papel clave que tiene el tipo de cambio real en una economía.

\subsection{Tipo de cambio real basado en los costos relativos unitarios laborales e índices relativos de precios al consumidor estimados por la OCDE}

A partir de 1970 la Organización para la Cooperación y el Desarrollo Económico (OCDE) publica periódicamente dos índices de tipo de cambio real efectivo para diversos países, entre ellos México, los cuales toman en cuenta no sólo los cambios en el mercado de divisas, sino también variaciones en los niveles de precios relativos. La OCDE utiliza dos índices de precios relativos para calcular el tipo de cambio real efectivo: los precios al consumidor y los costos unitarios laborales en manufacturas. ${ }^{6}$

En términos generales, podríamos decir que el cambio relativo en el índice de precios al consumidor entre dos periodos se obtiene comparando el cambio en el índice de precios al consumidor del propio país (convertido en dólares estadounidenses) con respecto a un promedio ponderado de los cambios en los índices de precios al consumidor de los países competidores. Los cambios relativos en el índice de costos unitarios laborales ${ }^{7}$ en manufacturas son calculados de la misma forma.

Algebraicamente el diferencial de precios usado por la OCDE para medir el tipo de cambio real definido como una medida de competitividad internacional, para el país $i$ es de la siguiente manera:

$$
\ln C_{i}=\ln P_{i}-\sum_{j \neq i} \ln \left(w_{i j} * P_{j}\right)
$$

\footnotetext{
${ }^{6}$ Por practicidad llamaremos a los índices publicados por la OCDE: TCR-ULC ${ }_{\text {OCDE }}$ al índice de tipo de cambio real efectivo basado en costos unitarios labores y TCR-CPI ${ }_{\mathrm{OCDE}}$ al índice de tipo de cambio real efectivo basado en los precios al consumidor

${ }^{7}$ Los costos unitarios laborales (ULC) miden el costo promedio de trabajo por unidad de producción y son calculados como la tasa de los costos laborales totales sobre la producción real.
} 
donde $P_{i}$ es el índice de precios (o costos) en dólares del país $i$ y $w_{i j}$ es un patrón de ponderación.

Los índices en dólares pueden ser reescritos como:

$$
P_{i}=P l_{i} * E_{i}
$$

$P l_{i}$ es el precio expresado en la moneda del país $i$ y $E_{i}$ el tipo de cambio con respecto al dólar. La ecuación (2.3) puede entonces ser reescrita como:

$$
\begin{gathered}
\ln C_{i}=\ln \left(P l_{i} * E_{i}\right)-\sum_{j \neq i} w_{i j} * \ln \left(P l_{j} * E_{j}\right) \\
\ln C_{i}=\left[\ln P l_{i}-\sum_{j \neq i} w_{i j} * \ln P l_{j}\right]+\left[\ln E_{i}-\sum_{j \neq i} w_{i j} * \ln E_{j}\right]
\end{gathered}
$$

Por lo tanto,

$$
C_{i}=\frac{E_{i} / \prod_{j \neq i} w_{i j} * E_{j}}{\prod_{j \neq i} P l_{j} w_{i j} / P l_{i}}
$$

$C_{j}$ es por definición el tipo de cambio real efectivo para el país $i$ donde $P l$ es usado como el índice de precios deflactor. Es decir, si $P l$ es el índice de costos unitarios laborales, tendría:

$$
T C R-U L C i_{O C D E}=\frac{E_{i} / \prod_{j \neq i} w_{i j} * E_{j}}{\prod_{j \neq i} U L C_{j} w_{i j} / U L C_{i}}
$$

$U L C_{i}$ y $U L C l_{j}$ son los índices de costos unitarios laborales en manufacturas para el país $i$ y $j$, respectivamente.

A su vez, para obtener el índice de tipo de cambio real efectivo basado en los índices de precios al consumidor tendríamos:

$$
T C R-C P I i_{O C D E}=\frac{E_{i} / \prod_{j \neq i} w_{i j} * E_{j}}{\prod_{j \neq i} P l_{j} w_{i j} / P l_{i}}
$$

donde $P l_{i}$ y $P l_{j}$ son los índices de precios al consumidor para el país $i$ y $j$, respectivamente. 
El esquema de ponderación $W_{i j}^{8}$, que se asume refleja la importancia relativa del competidor $j$ en el país $i$. Esta ponderación es medida a través de la suma de la parte de las importaciones del país $i$ ofertadas por el país $j\left(\mathrm{M}_{\mathrm{ij}}\right)$ sobre todos los $i$ mercados, multiplicado por la parte del total de las exportaciones del país $i$ hacia los $k$ 's mercados $\left(\mathrm{X}_{\mathrm{ki}}\right)$. Algebraicamente podríamos expresar lo anterior como:

$$
w_{i j}=\sum_{i \neq j} \frac{X_{k i}}{X_{k}} * \frac{M_{i j}}{M_{i}}
$$

\subsection{Tipo de cambio real basado en el índice de precios al por mayor y derechos especiales de giro (TCR-SDRWPI)}

Este índice de tipo de cambio real es llamado por Harberger (1988 pp. 181) como "Índice de Precios al por Mayor basado en Derechos Especiales de Giro" (SDRWPI), ${ }^{9}$ porque se usan las ponderaciones que el Fondo Monetario Internacional emplea en el cálculo de los derechos especiales de giro (DEG).

Algebraicamente el índice SDR-WPI es definido como el promedio ponderado de los WPIs de los cinco principales países comerciales (expresado en dólares), esto es:

$$
S D R W P I=S D R_{E U A} W I_{E U A}+\frac{S D R_{A L E} W P_{A L E}}{E_{A L E}}+\frac{S D R_{J P N} W P I_{I P N}}{E_{J P N}}+\frac{S D R_{R U} W I_{R U}}{E_{R U}}+\frac{S D R_{F R A} W P I_{F R A}}{E_{F R A}}
$$

$S D R_{E U A}, S D R_{A L E}, S D R_{J P N}, S D R_{R U}$ y $S D R_{F R A}$ representan la ponderación empleada por el FMI en el cálculo de los derechos especiales de giro para Estados Unidos, Alemania, Japón, Reino Unido y Francia, respectivamente; y donde $W P I_{E U A}, W P I_{A L E}, W P I_{J P N}, W P I_{R U}$ y $W P I_{F R A}$ son los índices de precios al por mayor de esos países, expresados en sus respectivas monedas.

\footnotetext{
${ }^{8}$ Para una explicación más detallada de este esquema de ponderación véase Durand (1986) y Maciejewski (1983).

${ }^{9}$ SDR son las siglas en inglés correspondientes a Special Drawing Rights, que son los derechos especiales de giro, y WPI son las siglas en inglés correspondientes a Wholesale Price Index, que es el indicador (índice) de precios al por mayor.
} 
Finalmente, $E$ representa el tipo de cambio nominal para cada país (expresado en unidades de moneda doméstica por dólar).

Alemania y Francia presentan una complicación adicional al construir el SDRWPI: en enero de 1999 estos países "renunciaron" a sus monedas domésticas por el euro, razón por la que el Fondo Monetario Internacional comenzó a reportar para ellos el tipo de cambio entre el euro y el dólar. Sin embargo, el FMI sigue presentando los índices de precios en las monedas originales. Para resolver esto Harberger (2005) propone convertir los índices de precios expresados en moneda doméstica a dólares usando el factor fijo para convertir las monedas nacionales en euros: para Alemania 1.95583 marcos por euro y para Francia 6.55957 francos por euro.

Adicionalmente, debido a los cambios en las valoraciones de las ponderaciones de las monedas en la cesta de $\mathrm{DEG}^{10}$, Harberger (2005) ha propuesto suavizar la transición de un esquema de ponderación a otro a través de una media móvil de 24 meses de las ponderaciones, comenzando 11 meses antes del periodo $t$ y terminando 12 meses después de $t$, donde $t$ es el mes en el que se dio oficialmente el cambio en la ponderación.

Una vez que hemos definido el deflactor de precios externos y usando el CPI como deflactor doméstico, el cálculo del TCR basado en SDRW$P I$ para el país $i$ se realiza de la siguiente forma:

$$
T C R_{i t}=E_{i t} \frac{S D R W P I_{i t}}{C P I_{i t}}
$$

dónde $C P I_{i t}$ es el índice de precios al consumidor del país $i$.

La razón por la que se incluye esta metodología en el trabajo es porque al haber definido el TCR como el precio que equilibra la balanza de pagos necesitamos un indicador que logre captar las variables que puedan provocar movimientos temporales o permanentes en la oferta y demanda reales de divisas. En este sentido, el TCR definido a través del SDRWPI es una manera muy simple de entender y calcular el índice de TCR y resulta un indicador adecuado para captar los movimientos de las variables que consideramos "fundamentos del TCR".

\footnotetext{
${ }^{10}$ Las ponderaciones en la valuación de la cesta de monedas DEG son revisadas por el FMI cada 5 años.

${ }^{11}$ Tales como flujos de capitales, liberalización comercial, cambios en el precio de un bien
} 
Además de lo anterior, Guillermo (2000) ofrece argumentos sólidos y algunos resultados empíricos que demuestran que, a pesar de la simplicidad de su cálculo (lo cual resulta ser una virtud en lugar de un defecto), el TCR basado en SDRWPI es una medida adecuada, e incluso mejor (en relación con las que se le compara) para la variable de nuestro interés, que es el tipo de cambio real.

\section{COMPARACIÓN ECONOMÉTRICA}

Después de haber presentado diferentes metodologías de medición correspondientes a indicadores alternativos para el tipo de cambio real es necesario hacer una comparación entre ellos, para saber cuál puede ser considerado el más adecuado para medir el tipo de cambio real ${ }^{12}$ para México. Para ello, las técnicas econométricas serán la herramienta que nos proporcione elementos en favor de una u otra medida alternativa del tipo de cambio real.

Como ya se mencionó, el tipo de cambio real es el precio real que hace que la oferta y la demanda reales de divisas se encuentren en equilibrio, por lo que podemos decir que, teóricamente, cambios en la oferta real de exportaciones y de la demanda real de importaciones deberán reflejar variaciones en el tipo de cambio real. Otra variable importante que afecta al tipo de cambio real es la entrada y salida de capitales, las cuales producen movimientos a lo largo de las curvas de oferta real de exportaciones y de demanda real de importaciones.

El análisis previo nos lleva a concluir que, empíricamente, una buena medida del tipo de cambio real debe ser sensible a cambios en la oferta de exportaciones, demanda de importaciones y de flujos de capital ${ }^{13}$. Tal es la razón por la que Guillermo (2000) propone el siguiente modelo econométrico para explicar los movimientos sobre el TCR, mismo que será utilizado en el presente trabajo para evaluar las medidas de TCR descritas con anterioridad:

$$
\operatorname{rer}_{t}=\propto_{0}+\propto_{1} \operatorname{rer}_{t-1}+\propto_{2} x g_{t-1}+\propto_{3} m g_{t}+\propto_{4} X M G_{t}+u_{t}
$$

clave en el comercio exterior de un país, etc.

${ }^{12}$ Los índices de tipo de cambio real utilizados en este análisis se presentan en los cuadros A1 y A2 del anexo, tanto para datos anuales como trimestrales.

${ }^{13}$ Para un desarrollo teórico de cómo estas variables afectan al tipo de cambio real véase Harberger (2004 y 2010). 
Dónde: $r e r_{t}$ es el logaritmo del índice de TCR en el tiempo $t ; x g_{t-1}$ es el logaritmo de la tasa de exportaciones (de bienes y servicios) con respecto al PIB en el periodo $t-1 ; m g_{t}$ es el logaritmo de la tasa de importaciones (de bienes y servicios) con respecto al PIB el tiempo $t$; y $X M G_{t}$ es la tasa de flujos de capital con respecto al PIB (definido como la diferencia entre las exportaciones e importaciones) en el tiempo $t$.

El propósito de la regresión de la ecuación (3.1), como indica Guillermo (2000), es saber que tan bien las definiciones de TCR usadas como variable dependiente están capturando el efecto de cambios en las variables explicativas. Ya que se pretende hacer una comparación de los resultados de las regresiones para cada una de las diferentes medidas de TCR, el análisis se realizará enfocándonos en la medida de bondad de ajuste $\left(R^{2}\right)$.

De esta manera, la regresión cuya $R^{2}$ sea más cercana a 1 será aquella en la que las variaciones del tipo de cambio real —utilizado como variable dependiente - son mejor explicadas por las variaciones de las variables que hemos definido como explicativas. A pesar de que la $R^{2}$ es un buen indicador para nuestro propósito es probable que ésta no sea comparable entre las regresiones, debido a que las variables dependientes no son construidas de la misma manera. Por ello se reportará lo que se conoce como la $R^{2}$ generalizada ${ }^{14}$ que nos permitirá tener una mejor conclusión sobre qué modelo presenta una mayor medida de bondad de ajuste.

Antes de correr la regresión (3.1) es necesario hacer un análisis sobre las características estacionarias o no estacionarias de las series utilizadas en el procedimiento de la estimación. Se realizaron pruebas Dickey-Fuller Aumentada (ADF) para todas las series con diferentes números de rezagos para datos trimestrales de 1970q1 a 2011q3 para las variables del tipo de cambio real $^{15}$ y de $1981 \mathrm{q} 1$ a $2011 \mathrm{q} 3$ para las variables independientes; y para datos anuales de 1970 a 2010 para todas las variables. Debido a que un proceso estocástico puede incluir o excluir un término constante y

\footnotetext{
${ }^{14}$ Recordemos que la $R^{2}$ generalizada se define como el cuadrado de la correlación entre la $y$ observada y la $y$ estimada. Es decir:

$$
R_{g}^{2}=(\operatorname{Corr}(y, \hat{y}))^{2}=\frac{[\operatorname{Cov}(y, \hat{y})]^{2}}{\operatorname{Var}(y) \operatorname{Var}(\hat{y})}
$$

${ }^{15}$ Excepto el tipo de cambio real publicado por el FMI, cuyo análisis es de 1980 q1 a $2011 \mathrm{q} 3$.
} 
puede incluir o excluir una tendencia en el tiempo. Se realizaron las tres variaciones de la prueba $\mathrm{ADF}$ para tomar en cuenta el papel que pudiera tener el término constante y la tendencia en nuestras variables de interés.

Los resultados de la pruebas ADF nos indica que existe suficiente evidencia para afirmar que las diferentes definiciones de tipo de cambia real y las variables explicativas (exportaciones como proporción del PIB en un rezago, importaciones como proporción del PIB y flujos de capital como proporción del PIB) tienen un comportamiento de caminata aleatoria.

$\mathrm{Al}$ encontrar que todas las variables utilizadas en nuestro modelo son series no estacionarias procederemos a encontrar su orden de integración. De nuevo se aplicaron pruebas ADF, tanto para las diferentes definiciones de tipo de cambio real como para las variables explicativas —en datos anuales y trimestrales - , sólo que, a diferencia de las pruebas anteriores, todas las variables son tomadas en primeras diferencias. El resultado que podemos apreciar es que, en todas las variables, se rechaza la hipótesis de no estacionariedad para las diferentes pruebas ADF, por lo que podemos afirmar que las variables en su conjunto son integradas de orden uno $(\mathrm{I}(1)){ }^{16}$

Como mencionan Hill, Griffiths y Lim (2011), ${ }^{17}$ las variables de series de tiempo no estacionarias no deberían ser utilizadas en modelos de regresión para evitar el problema de regresiones espurias. No obstante, hay una excepción para esta regla. Si $y_{t}$ y $x_{t}$ son variables no estacionarias e integradas de orden 1 hay la posibilidad de que la relación lineal existente entre ellas arroje un término de error $e_{t}=y_{t}-\beta_{1}-\beta_{2} x_{t}$, que sea un proceso estacionario $\mathrm{I}(0)$. En este caso decimos que $y_{t}$ y $x_{t}$ están cointegradas.

Debido a lo anterior es importante averiguar si la relación entre las variables presentadas en la regresión (3.1) es verdadera o espuria. Un modo natural de saber si la relación entre nuestras variables está cointegrada es probar si los errores que producen son estacionarios. Ya que no podemos observar $e_{t}$, estimamos la regresión (3.1) por mínimos cuadrados y obtenemos los residuales estimados, es decir:

$$
\widehat{u_{t}}=r e r_{t}-\widehat{\alpha_{0}}-\widehat{\alpha_{1}} r e r_{t-1}-\widehat{\alpha_{2}} x g_{t-1}-\widehat{\alpha_{3}} m g_{t}-\widehat{\alpha_{4}} X M G_{t}
$$

\footnotetext{
${ }^{16}$ Recordemos que si $y_{t}$ sigue una caminata aleatoria, entonces $\gamma=0$ y la primera diferencia de $y_{t}$ se vuelve $\Delta y_{t}=y_{t}-y_{t-1}=v_{t} ; \mathrm{y}$, ya que $v_{t}$ es una variable aleatoria independiente $\left(0, \sigma_{y}^{2}\right)$ es estacionaria, por lo que decimos que $y_{t}$ es integrada de orden 1 .

${ }^{17}$ Hill, Griffiths y Lim (2011), pág. 488.
} 
Para probar la estacionariedad en el término de error utilizaremos la prueba $\mathrm{ADF}$ para diferentes números de rezagos y restringiremos el término constante a cero. Los resultados de la prueba de cointegración hecha para las cinco diferentes medidas de TCR usadas como variable dependiente indican que, en general, no se encuentra evidencia (con datos anuales y trimestrales) en ninguna de las regresiones de que las variables estén cointegradas. Por lo que podemos concluir que la relación expresada en la ecuación (3.1) no es válida para relacionar al TCR con las variables que hemos definido como su fundamento. ${ }^{18}$

El hecho de que no encontráramos evidencia de cointegración en la relación entre las variables para las diferentes medidas de tipo de cambio real nos lleva a plantear la especificación de un modelo que tome en cuenta el comportamiento I(1) de nuestras variables. La especificación de este modelo simplemente implica que tomemos las primeras diferencias de cada una de las series (variables dependientes e independientes), procedimiento que resultará en un término de error estacionario y, por lo tanto, en errores estándar válidos para ser utilizados en inferencia respecto a los coeficientes de la regresión de interés.

De esta forma, si seguimos de nuevo a Guillermo (2000) el modelo propuesto es expresado de la siguiente manera:

$$
\Delta r e r_{t}=\propto_{0}+\propto_{1} \Delta X G_{t-1}+\propto_{2} \Delta M G_{t}+\propto_{3} \Delta X M G_{t}+u_{t}
$$

donde $\Delta r r_{t}$ es la primera diferencia del logaritmo del TCR en el tiempo $t$; $\Delta X G_{t-1}$ es el cambio en la tasa de exportaciones con respecto al PIB en el tiempo $t-1 ; \Delta M G_{t}$ es el cambio en la tasa de importaciones con respecto al PIB en el tiempo $t$ y $\triangle X M G_{t}$ es el cambio en la tasa de flujos de capital con respecto al PIB en el tiempo $t$.

Es necesario mencionar aquí que las primeras diferencias tomadas como variables explicativas en (3.2) son las primeras diferencias de la tasa de exportaciones con respecto al PIB; y las primeras diferencias de la tasa de importaciones con respecto al PIB, en lugar de las diferencias de los logaritmos de estas tasas (que son aproximadamente igual al cambio porcentual de ellas). La razón de hacer esto es que tiene mayor sentido económico tomar la

\footnotetext{
${ }^{18}$ Por motivos de espacio no se presentan los resultados de las pruebas de estacionariedad y de cointegración, pero pueden ser solicitados al autor.
} 
primera diferencia de las tasas en vez de sus cambios porcentuales. Como explica Guillermo (2000):

"Tomar las primeras diferencias de las tasas tiene más sentido económico porque de esta manera podemos asegurar que el efecto de un movimiento, por ejemplo, de la tasa de importaciones con respecto al PIB, de 1 a $2 \%$ es "igual" al efecto de un movimiento en esta tasa de 5 a $6 \%$, y no "igual" a un movimiento de 5 a 10\%" (pág. 148, traducción propia).

El análisis de los resultados de la estimación de la ecuación (3.2), utilizando las diferentes metodologías de medición correspondientes a los cinco indicadores para el tipo de cambio real con una muestra anual de 1970 a 2010, que se presentan en el cuadro 1, puede ser resumido de la siguiente manera:

El modelo en su conjunto es significativo para las cinco regresiones.

El modelo en que se toma la medida de tipo de cambio real basada en el SDRWPI muestra tanto un $R^{2}$ como $\mathrm{R}^{2}$ generalizado más altos, en relación con las otras cuatro medidas presentadas.

En todos los casos los signos de los coeficientes son los esperados por la teoría: un incremento en las exportaciones provocará una caída en el tipo de cambio real; un incremento en las importaciones llevará a una depreciación del tipo de cambio real; y una entrada de capital apreciará al tipo de cambio real.

Para el modelo basado en el SDRWPI y para el modelo basado en la medida del FMI, todos los coeficientes relativos a las variables fundamentos del TCR son estadísticamente significativos; el coeficiente asociado a la variable $\Delta \mathrm{xg}_{\mathrm{t}-1}$ no es significativo ${ }^{19}$ en los modelos que toman las medidas del Banco de México y en los

\footnotetext{
${ }^{19}$ Debemos tener en cuenta aquí que la insignificancia estadística de estos coeficientes podría deberse a la presencia de colinealidad. No obstante, este problema no afecta la conclusión respecto a qué definición de TCR podría ser mejor.
} 
de la OCDE; para la variable $\Delta \mathrm{mg}_{\mathrm{t}}$ su coeficiente asociado no es significativo para ambas medidas del TCR presentadas por la OCDE; finalmente, el coeficiente asociado a la variable $\Delta \mathrm{XMG}_{\mathrm{t}}$ no es significativo para la medida presentada por el Banco de México.

\section{Cuadro 1}

Resultados de la estimación para diferentes medidas del TCR para México Datos anuales de 1970 a 2010

Ecuación estimada: $\Delta \operatorname{rer}_{\mathrm{t}}=\alpha_{0}+\alpha_{1} \Delta X G_{\mathrm{t}-1}+\alpha_{2} \Delta M G_{2}+\alpha_{3} \Delta X M G_{\mathrm{t}}+u_{\mathrm{t}}$

\begin{tabular}{|c|c|c|c|c|c|c|c|c|c|}
\hline ITCR & Variables & Coeficiente & $\begin{array}{c}\text { Error } \\
\text { estandar }\end{array}$ & $\begin{array}{c}\text { Estadístico } \\
t\end{array}$ & $P$-value & $R^{2}$ & $R_{g}^{2}$ & $\begin{array}{c}\text { Estadístico } \\
F\end{array}$ & $\begin{array}{c}P \text {-value } \\
\text { (F) }\end{array}$ \\
\hline \multirow{4}{*}{ TCR-BX } & Constante & -0.0048 & 0.0213 & -0.23 & 0.823 & 0.58 & 0.583 & 11.66 & 0.000 \\
\hline & $\Delta \mathrm{xg}_{\mathrm{t}-1}$ & -1.1987 & 0.6267 & -1.91 & 0.067 & & & & \\
\hline & $\Delta \mathrm{mg}_{\mathrm{t}}$ & 3.1215 & 1.0552 & 2.96 & 0.007 & & & & \\
\hline & $\Delta \mathrm{XMG}_{\mathrm{t}}$ & -3.8530 & 0.7653 & -0.23 & 0.823 & & & & \\
\hline \multirow{4}{*}{ TCR-FMI } & Constante & 0.0001 & 0.0172 & 0.01 & 0.993 & 0.68 & 0.682 & 17.93 & 0.000 \\
\hline & $\Delta \mathrm{xg}_{\mathrm{t}-1}$ & -1.2057 & 0.5080 & -2.37 & 0.026 & & & & \\
\hline & $\Delta \mathrm{mg}_{\mathrm{t}}$ & 2.3718 & 0.8553 & 2.77 & 0.010 & & & & \\
\hline & $\Delta \mathrm{XMG}_{\mathrm{t}}$ & -4.1476 & 0.6203 & -6.69 & 0.000 & & & & \\
\hline \multirow{4}{*}{$\begin{array}{c}\text { TCR- } \\
\text { RELCPI }_{\text {OCDE }}\end{array}$} & Constante & 0.0050 & 0.0183 & 0.27 & 0.787 & 0.68 & 0.689 & 18.52 & 0.000 \\
\hline & $\Delta \mathrm{xg}_{\mathrm{t}-1}$ & -0.8108 & 0.5404 & -1.50 & 0.146 & & & & \\
\hline & $\Delta \mathrm{mg}_{\mathrm{t}}$ & 0.8771 & 0.9098 & 0.96 & 0.344 & & & & \\
\hline & $\Delta \mathrm{XMG}_{\mathrm{t}}$ & -4.8749 & 0.6598 & -7.39 & 0.000 & & & & \\
\hline \multirow{4}{*}{$\begin{array}{c}\text { TCR- } \\
\text { RELULC }_{\text {OCDE }}\end{array}$} & Constante & 0.0039 & 0.0217 & 0.18 & 0.858 & 0.66 & 0.668 & 16.83 & 0.000 \\
\hline & $\Delta \mathrm{xg}_{\mathrm{t}-1}$ & -0.1359 & 0.6408 & -0.21 & 0.834 & & & & \\
\hline & $\Delta \mathrm{mg}_{\mathrm{t}}$ & 1.3174 & 1.0790 & 1.22 & 0.233 & & & & \\
\hline & $\Delta \mathrm{XMG}_{\mathrm{t}}$ & -5.4512 & 0.7825 & -6.97 & 0.000 & & & & \\
\hline \multirow{4}{*}{$\begin{array}{c}\text { TCR- } \\
\text { SDRWPI }\end{array}$} & Constante & -0.0095 & 0.0168 & -0.57 & 0.574 & 0.71 & 0.714 & 20.84000 & 0.0000 \\
\hline & $\Delta \mathrm{xg}_{\mathrm{t}-1}$ & -1.4535 & 0.4946 & -2.94 & 0.007 & & & & \\
\hline & $\Delta \mathrm{mg}_{\mathrm{t}}$ & 2.8368 & 0.8327 & 3.41 & 0.002 & & & & \\
\hline & $\Delta \mathrm{XMG}_{\mathrm{t}}$ & -4.1840 & 0.6039 & -6.93 & 0.000 & & & & \\
\hline
\end{tabular}

Fuente: Estimación propia. 
Los resultados de la estimación de la ecuación (3.2) usando una muestra con datos trimestrales de 1981q1 a 2011q3 se presentan en el cuadro 2 y pueden ser resumidas de la siguiente manera:

Igual que en el caso de los datos anuales, el modelo en su conjunto es significativo para las cinco regresiones.

Nuevamente el modelo en que se toma la medida de tipo de cambio real basada en el SDRWPI muestra tanto un $R^{2}$ como $R^{2}$ generalizado más altos en relación con las otras cuatro medidas presentadas.

En todos los casos los signos de los coeficientes son los esperados por la teoría presentada en el capítulo I: un incremento en las exportaciones provocará una caída en el tipo de cambio real, un incremento en las importaciones llevará a una depreciación del tipo de cambio real y una entrada de capital apreciará al tipo de cambio real.

El coeficiente $\Delta \mathrm{xg}_{\mathrm{t}-1}$ no es significativo en todos los modelos, los coeficientes asociados a las variables $\Delta \mathrm{mg}_{\mathrm{t}}$ y $\Delta \mathrm{XMG}_{\mathrm{t}}$ son estadísticamente significativos en todos los casos.

Si tomamos los resultados de la estimación de la ecuación (3.2) tanto para la muestra anual como para la trimestral podemos concluir que, para México, el mejor indicador para medir el tipo de cambio real es el basado en el SDRWPI. Debemos recordar que el término "mejor" debe ser entendido aquí en el sentido de que las variables independientes (las cuales definimos teóricamente como determinantes del tipo de cambio real) explican de mejor forma y de manera conjunta los movimientos del TCR, comparados con los que estas mismas variables explican los movimientos de las otras medidas del tipo de cambio real. 


\section{Cuadro 2}

Resultados de la estimación para diferentes medidas del TCR para México.

Datos trimestrales de 1981q1 a 2011q3

Ecuación estimada: $\Delta r e r_{\mathrm{t}}=\alpha_{0}+\alpha_{1} \Delta X G_{\mathrm{t}-1}+\alpha_{2} \Delta M G_{2}+\alpha_{3} \Delta X M G_{\mathrm{t}}+u_{\mathrm{t}}$

\begin{tabular}{|c|c|c|c|c|c|c|c|c|c|}
\hline ITCR & Variables & Coeficiente & $\begin{array}{c}\text { Error } \\
\text { estándar }\end{array}$ & $\begin{array}{c}\text { Estadístico } \\
t\end{array}$ & P-value & $R^{2}$ & $R_{g}^{2}$ & $\begin{array}{c}\text { Estadístico } \\
\qquad F\end{array}$ & $\begin{array}{c}P \text {-value } \\
\text { (F) }\end{array}$ \\
\hline \multirow{4}{*}{ TCR-BX } & Constante & -0.0008 & 0.00696 & -0.13 & 0.900 & 0.276 & 0.276 & 14.89 & .000 \\
\hline & $\Delta \mathrm{xg}_{\mathrm{t}-1}$ & -0.0653 & 0.40925 & -0.16 & 0.873 & & & & \\
\hline & $\Delta \mathrm{mg}_{\mathrm{t}}$ & 8.5316 & 1.72651 & 4.94 & 0.000 & & & & \\
\hline & $\Delta \mathrm{XMG}_{\mathrm{t}}$ & -3.0979 & 0.51516 & -6.01 & 0.000 & & & & \\
\hline \multirow{4}{*}{ TCR-FMI } & Constante & -0.0011 & 0.00454 & -0.25 & 0.804 & 0.504 & 0.504 & 39.7 & .000 \\
\hline & $\Delta \mathrm{xg}_{\mathrm{t}-1}$ & -0.2643 & 0.26716 & -0.99 & 0.325 & & & & \\
\hline & $\Delta \mathrm{mg}_{\mathrm{t}}$ & 9.5824 & 1.12709 & 8.5 & 0.000 & & & & \\
\hline & $\Delta \mathrm{XMG}_{\mathrm{t}}$ & -3.2288 & 0.33630 & -9.6 & 0.000 & & & & \\
\hline \multirow{4}{*}{$\begin{array}{l}\text { TCR- } \\
\text { RELC- } \\
\text { PI }_{\text {OCDE }}\end{array}$} & Constante & 0.0001 & 0.00692 & 0.03 & 0.979 & 0.277 & 0.277 & 15 & .000 \\
\hline & $\Delta \mathrm{xg}_{\mathrm{t}-1}$ & -0.7926 & 0.40712 & -1.95 & 0.054 & & & & \\
\hline & $\Delta \mathrm{mg}_{\mathrm{t}}$ & 7.8489 & 1.71753 & 4.57 & 0.000 & & & & \\
\hline & $\Delta \mathrm{XMG}_{\mathrm{t}}$ & -3.1759 & 0.51248 & -6.2 & 0.000 & & & & \\
\hline \multirow{4}{*}{$\begin{array}{c}\text { TCR- } \\
\text { RELUL- } \\
\mathrm{C}_{\mathrm{OCDE}}\end{array}$} & Constante & 0.0003 & 0.00831 & 0.05 & 0.963 & 0.263 & 0.263 & 13.98 & 0.000 \\
\hline & $\Delta \mathrm{xg}_{\mathrm{t}-1}$ & -0.2821 & 0.48866 & -0.58 & 0.565 & & & & \\
\hline & $\Delta \mathrm{mg}_{\mathrm{t}}$ & 6.9768 & 2.06154 & 3.38 & 0.001 & & & & \\
\hline & $\Delta \mathrm{XMG}_{\mathrm{t}}$ & -3.9074 & 0.61513 & -6.35 & 0.000 & & & & \\
\hline \multirow{4}{*}{$\begin{array}{l}\text { TCR- } \\
\text { SDRWPI }\end{array}$} & Constante & -0.0032 & 0.00440 & -0.74 & 0.4590 & 0.536 & 0.535 & 45.06 & 0.0000 \\
\hline & $\Delta \mathrm{xg}_{\mathrm{t}-1}$ & -0.1227 & 0.25872 & -0.47 & 0.6360 & & & & \\
\hline & $\Delta \mathrm{mg}_{\mathrm{t}}$ & 10.6284 & 1.09149 & 9.74 & 0.0000 & & & & \\
\hline & $\Delta \mathrm{XMG}_{\mathrm{t}}$ & -3.1324 & 0.32568 & -9.62 & 0.0000 & & & & \\
\hline
\end{tabular}

Fuente: Estimación propia. 


\section{CONCLUSIONES}

En este trabajo se presentaron cinco diferentes metodologías para la medición del tipo de cambio real: la calculada por el Banco de México, la del FMI, dos publicadas por la OCDE y la propuesta por Harberger denominada SDRWPI. Se pudo apreciar claramente que existen grandes diferencias en la forma en cada una de estas alternativas de medición del TCR son construidas. Específicamente, las medidas del TCR estimadas por el FMI y la OCDE parecen enfocarse en ser una indicador de competitividad, lo cual, como ya vimos, no es el papel clave que desempeña el tipo de cambio real en una economía, además de que el uso de indicadores de competitividad expresados como un índice en una año base debe ser manejado con cautela para hacer comparaciones entre países.

Por otro lado, el índice publicado por el Banco de México pone más énfasis en la importancia que un país tiene sobre el PIB mundial, más que en la que dicho país tenga en el flujo comercial internacional o en la importancia relativa de los países en los flujos comerciales con México.

Debido a lo anterior fue realizada una comparación econométrica de las cinco medidas alternativas del tipo de cambio real, arriba mencionadas, con el objetivo de tener un soporte empírico que nos pueda decir cuál de estos índices de TCR puede ser preferido en relación con los otros. El objetivo del análisis econométrico —una vez definida la relación adecuada entre las variables - fue el de saber que tan bien los cambios en el TCR son explicados por cambios en las variables explicativas, por lo que el mejor indicador del TCR será aquel en el que las variables independientes (las cuales definimos teóricamente como determinantes del tipo de cambio real), explican de mejor forma y de manera conjunta los movimientos del TCR. En este sentido, la medida de bondad de ajuste $R^{2}$, la significancia de los coeficientes y la significancia general del modelo (estadístico F) fueron comparados en las cinco definiciones.

El resultado arrojado por el análisis econométrico en este trabajo sugiere que la medida del tipo de cambio real en México basado en el SDRWPI es mejor que las otras cuatro con las que se le compara. Por esta razón podríamos decir que el TCR basado en el SDRWPI es un índice apropiado para ser entendido como un indicador del TCR, en el sentido de que nos da una adecuada medida para México del precio real que equilibra la balanza de pagos. 


\section{BIBLIOGRAFÍA}

Bayoumi, T., J. Lee y S. Jayanthi. 2005. New Rates from New Weights, IMF Working Paper 05/97.

Carballo, E. y C.M. Urzúa. 2008. "Un nuevo índice de tipo de cambio real para México", Comercio Exterior, vol. 58, núms. 8-9, agostoseptiembre, 611-616.

Duran, Martine. 1986. Method of Calculating Effective Exchange Rate and Indicators of Competitiveness, OECD Economics Department Working Papers, núm. 29. Guillermo Peón, S.B. 2000. A Theoretical and Empirical Analysis on Real Exchange Rate Behavior and Measurement, University of California, Los Angeles, Ph.D. Dissertation Work.

------. 2003. "El tipo de cambio real: teoría y evidencia empírica", Problemas del Desarrollo, XXXIV( 132): 9-29.

Harberger, A. 1988. "Trade Policy and the Real Exchange Rate: Some Theoretical and Practical Applications for Developing Countries", The Economic Development Institute of the World Bank.

Harberger, Arnold. 2004 "The Real Exchange Rate: Issues of Concept and Measurement, Paper Prepared for a Conference in Honor of Michael Mussa". University of California, Los Angeles.

Harberger, Arnold. 2005. "On the Process of Growth and Economic Policy in Developing Countries", PPC Issue Paper No. 13, USAID.

Harberger, Arnold. 2010. "Real Exchange Rate Adjustment versus "Standard" Inflation: The Case of Russia. 2000-2010”, University of California, Los Angeles. Hill, C; W. Griffiths, y G. Lim. 2011. Principles of Econometrics, John Wiley \& Sons.

Maciejewski, E.B. 1983. "Real Effective Exchange Rate Indices: A ReExamination of the Major Conceptual an Methodological Issues", Staff Papers - IMF,30(3): 491-541.

Zanello, Alessandro y D. Desruelle. 1997. “A Primer on the IMF's Information Notice System”, IMF Working Paper, núm. 97/71. 


\section{ANEXO}

\section{Cuadro A1}

Diferentes índices de tipo de cambio real.

Datos anuales de 1970 a 2010 (2005=100)

\begin{tabular}{|c|c|c|c|c|c|}
\hline Periodo & $\begin{array}{c}\text { Banco de } \\
\text { México }\end{array}$ & $\begin{array}{l}\text { Índices } \\
\text { relativos de } \\
\text { precios al } \\
\text { consumidor } \\
\quad(F M I)\end{array}$ & $\begin{array}{c}\text { Índice relativo } \\
\text { de precios al } \\
\text { consumidor } \\
\text { (OCDE) }\end{array}$ & $\begin{array}{c}\text { Costos relati- } \\
\text { vos unitarios } \\
\text { laborales } \\
\text { (OCDE) }\end{array}$ & SDRWPI \\
\hline 1970 & 88.8241631 & & 97.9184496 & 77.4698352 & 113.442975 \\
\hline 1971 & 89.877232 & & 98.2544121 & 77.3081309 & 111.738306 \\
\hline 1972 & 94.8514632 & & 100.610363 & 76.9186393 & 115.106632 \\
\hline 1973 & 97.5750191 & & 100.457917 & 78.9639924 & 123.46838 \\
\hline 1974 & 88.0045622 & & 90.3588058 & 72.6810212 & 118.596901 \\
\hline 1975 & 86.2159428 & & 86.5908073 & 67.2528877 & 109.89851 \\
\hline 1976 & 93.8403798 & & 91.5807105 & 67.1772135 & 118.757446 \\
\hline 1977 & 119.175596 & & 117.989283 & 90.2791884 & 148.01382 \\
\hline 1978 & 118.388571 & & 113.327161 & 91.918101 & 143.380122 \\
\hline 1979 & 111.400564 & & 107.20214 & 86.510803 & 140.151634 \\
\hline 1980 & 99.7263581 & 91.4160344 & 96.5157803 & 80.7640277 & 129.765054 \\
\hline 1981 & 84.2685869 & 80.6267385 & 84.6030846 & 77.3036487 & 107.925547 \\
\hline 1982 & 114.010743 & 110.991898 & 111.020543 & 110.341784 & 148.402848 \\
\hline 1983 & 129.370593 & 126.646403 & 172.665796 & 186.915888 & 159.421715 \\
\hline 1984 & 108.830819 & 108.009829 & 131.631999 & 156.690693 & 133.773457 \\
\hline 1985 & 106.317134 & 105.100897 & 139.390171 & 172.46583 & 126.230233 \\
\hline 1986 & 161.975034 & 150.806816 & 164.50212 & 203.925567 & 172.941624 \\
\hline 1987 & 168.262036 & 163.543441 & 170.518945 & 216.872696 & 188.541154 \\
\hline 1988 & 151.103422 & 131.947881 & 134.717547 & 180.407721 & 156.786029 \\
\hline 1989 & 136.620874 & 123.005012 & 126.672858 & 161.088961 & 143.247008 \\
\hline 1990 & 135.875484 & 120.266993 & 122.570079 & 156.653873 & 133.373247 \\
\hline 1991 & 124.248382 & 109.678186 & 110.679571 & 139.372822 & 116.507523 \\
\hline 1992 & 116.677627 & 101.3916 & 102.12677 & 124.443891 & 106.882775 \\
\hline 1993 & 99.9189842 & 94.2677361 & 95.5734214 & 114.200879 & 97.8665612 \\
\hline 1994 & 103.1642 & 97.8258211 & 99.971708 & 117.955826 & 104.521314 \\
\hline
\end{tabular}




\begin{tabular}{|l|r|r|r|r|r|}
\hline 1995 & 159.207884 & 146.309347 & 147.562772 & 189.906471 & 157.478809 \\
\hline 1996 & 139.931775 & 130.257802 & 132.161152 & 179.872291 & 131.510748 \\
\hline 1997 & 116.647112 & 113.143504 & 114.276873 & 150.755663 & 110.87121 \\
\hline 1998 & 114.833605 & 112.2765 & 113.210627 & 147.058824 & 103.999017 \\
\hline 1999 & 105.485044 & 103.434901 & 103.402789 & 127.169835 & 95.5536788 \\
\hline 2000 & 93.3243135 & 95.5163054 & 95.1777254 & 109.847861 & 88.5262547 \\
\hline
\end{tabular}

\section{Cuadro A1}

(Continuación)

\begin{tabular}{|c|c|c|c|c|c|}
\hline Periodo & $\begin{array}{c}\text { Banco de } \\
\text { México }\end{array}$ & $\begin{array}{c}\text { Índices } \\
\text { relativos de } \\
\text { precios al } \\
\text { consumidor } \\
\text { (FMI) }\end{array}$ & $\begin{array}{c}\text { Índice } \\
\text { relativo de } \\
\text { precios al } \\
\text { consumidor } \\
\text { (OCDE) }\end{array}$ & $\begin{array}{c}\text { Costos } \\
\text { relativos } \\
\text { unitarios } \\
\text { laborales } \\
\text { (OCDE) }\end{array}$ & SDRWPI* \\
\hline 2001 & 85.0884142 & 89.8082594 & 89.1836309 & 99.4777419 & 78.8247257 \\
\hline 2002 & 82.7419806 & 89.8001946 & 88.8692192 & 95.0660709 & 77.8487397 \\
\hline 2003 & 97.2764214 & 100.296711 & 99.5884011 & 104.305197 & 91.4650247 \\
\hline 2004 & 104.591629 & 104.325147 & 103.7667 & 104.474103 & 101.088559 \\
\hline 2005 & 100 & 100 & 100 & 100 & 100 \\
\hline 2006 & 98.7630463 & 99.7879506 & 99.9967001 & 99.3936984 & 103.687755 \\
\hline 2007 & 101.62361 & 100.88018 & 100.920048 & 99.2063492 & 108.200238 \\
\hline 2008 & 106.191992 & 102.555337 & 102.643912 & 105.769739 & 113.851422 \\
\hline 2009 & 120.366975 & 117.131451 & 117.038746 & 126.574267 & 118.018266 \\
\hline 2010 & 110.866369 & 107.848688 & 108.25237 & 116.64188 & 113.504689 \\
\hline
\end{tabular}

Fuente: Banco de México, IMF y OCDE.

* Estimación propia con datos del IMF. 


\section{Cuadro A2}

Diferentes índices de tipo de cambio real.

Datos trimestrales 1980q1 a 2011q3 (2005=100)

\begin{tabular}{|c|c|c|c|c|c|}
\hline Periodo & $\begin{array}{c}\text { Banco de } \\
\text { México }\end{array}$ & $\begin{array}{c}\text { Índices } \\
\text { relativos de } \\
\text { precios al } \\
\text { consumidor } \\
\text { (FMI) }\end{array}$ & $\begin{array}{c}\text { Índice } \\
\text { relativo de } \\
\text { precios al } \\
\text { consumidor } \\
\text { (OCDE) }\end{array}$ & $\begin{array}{c}\text { Costos } \\
\text { relativos } \\
\text { unitarios } \\
\text { laborales } \\
\text { (OCDE) }\end{array}$ & SDRWPI* \\
\hline 1980 Q1 & 102.79985 & 94.8586606 & 100.174464 & 83.0151087 & 136.652257 \\
\hline 1980 Q2 & 101.194255 & 93.6271144 & 98.2583704 & 79.3965859 & 130.705216 \\
\hline 1980 Q3 & 99.1276342 & 90.1523575 & 95.1452154 & 81.3802083 & 128.297076 \\
\hline 1980 Q4 & 95.7836935 & 87.4049471 & 92.816814 & 79.377679 & 123.405666 \\
\hline 1981 Q1 & 89.8183526 & 85.1426139 & 87.9463387 & 80.0448251 & 115.272858 \\
\hline 1981 Q2 & 84.4740532 & 81.0175808 & 84.5292103 & 83.5282325 & 109.153151 \\
\hline 1981 Q3 & 80.7940925 & 78.6348982 & 82.8850633 & 78.369906 & 104.019024 \\
\hline 1981 Q4 & 81.9878491 & 78.0863635 & 83.2369172 & 68.8752669 & 103.257155 \\
\hline
\end{tabular}

Cuadro A2

(Continuación)

\begin{tabular}{|c|c|c|c|c|r|}
\hline Periodo & $\begin{array}{c}\text { Banco de } \\
\text { México }\end{array}$ & $\begin{array}{c}\text { Índices } \\
\text { relativos de } \\
\text { precios al } \\
\text { consumidor } \\
\text { (FMI) }\end{array}$ & $\begin{array}{c}\text { Índice } \\
\text { relativo de } \\
\text { precios al } \\
\text { consumidor } \\
\text { (OCDE) }\end{array}$ & $\begin{array}{c}\text { Costos } \\
\text { relativos } \\
\text { unitarios } \\
\text { laborales } \\
\text { (OCDE) }\end{array}$ & SDRWPI* \\
\hline 1982 Q1 & 97.4392908 & 90.2255639 & 95.4783369 & 83.6120401 & 122.399457 \\
\hline 1982 Q2 & 113.919808 & 112.692987 & 117.312208 & 111.445447 & 142.687148 \\
\hline 1982 Q3 & 123.564929 & 133.994372 & 113.839982 & 119.502868 & 177.544138 \\
\hline 1982 Q4 & 121.118944 & 116.027228 & 121.252318 & 143.884892 & 150.98065 \\
\hline 1983 Q1 & 136.108862 & 136.208854 & 214.256912 & 276.395799 & 167.513306 \\
\hline 1983 Q2 & 131.176401 & 128.926898 & 179.223675 & 186.776242 & 161.211067 \\
\hline 1983 Q3 & 126.194572 & 123.45679 & 158.287607 & 162.601626 & 156.541991 \\
\hline 1983 Q4 & 124.002538 & 119.246363 & 151.478177 & 159.286397 & 152.420494 \\
\hline 1984 Q1 & 116.184309 & 116.2971 & 136.912425 & 151.860289 & 143.159922 \\
\hline 1984 Q2 & 112.155103 & 110.721535 & 138.582084 & 169.808117 & 137.939944 \\
\hline 1984 Q3 & 105.770495 & 105.392587 & 128.145951 & 160.076837 & 130.3079 \\
\hline
\end{tabular}




\begin{tabular}{|r|r|r|r|r|r|}
\hline 1984 Q4 & 101.213368 & 100.857287 & 124.003846 & 146.907595 & 123.686065 \\
\hline 1985 Q1 & 91.450987 & 95.9631502 & 118.845822 & 147.318798 & 112.23467 \\
\hline 1985 Q2 & 94.6859555 & 96.6121345 & 123.219494 & 155.448469 & 114.41299 \\
\hline 1985 Q3 & 112.089701 & 110.075585 & 152.455781 & 184.501845 & 132.474931 \\
\hline 1985 Q4 & 127.041891 & 121.911573 & 178.339396 & 219.683656 & 145.79834 \\
\hline 1986 Q1 & 140.781755 & 137.671516 & 155.288835 & 197.472354 & 155.812885 \\
\hline 1986 Q2 & 154.908049 & 147.152597 & 160.206397 & 190.730498 & 166.839722 \\
\hline 1986 Q3 & 171.390695 & 157.438992 & 169.320741 & 206.868018 & 180.970168 \\
\hline 1986 Q4 & 180.819637 & 163.585801 & 174.572289 & 223.513634 & 188.143722 \\
\hline 1987 Q1 & 186.90165 & 173.882803 & 172.951972 & 230.893558 & 192.838704 \\
\hline 1987 Q2 & 188.280876 & 170.71644 & 171.704741 & 226.449275 & 193.901538 \\
\hline 1987 Q3 & 120.434397 & 157.521659 & 163.962676 & 202.224469 & 183.866539 \\
\hline 1987 Q4 & 177.43122 & 153.814602 & 173.823804 & 210.43771 & 183.557834 \\
\hline 1988 Q1 & 166.476215 & 147.95088 & 148.626439 & 206.996481 & 170.534882 \\
\hline 1988 Q2 & 153.290813 & 134.680135 & 135.575977 & 183.587296 & 158.843801 \\
\hline 1988 Q3 & 141.377161 & 124.496825 & 128.679129 & 169.520258 & 148.467355 \\
\hline 1988 Q4 & 143.269499 & 123.477116 & 127.938295 & 166.805671 & 149.298077 \\
\hline
\end{tabular}

Cuadro A2

(Continuación)

\begin{tabular}{|c|r|r|r|r|r|}
\hline Periodo & $\begin{array}{c}\text { Banco de } \\
\text { México }\end{array}$ & $\begin{array}{c}\text { Índices } \\
\text { relativos de } \\
\text { precios al } \\
\text { consumidor } \\
\text { (FMI) }\end{array}$ & $\begin{array}{c}\text { Índice } \\
\text { relativo de } \\
\text { precios al } \\
\text { consumidor } \\
\text { (OCDE) }\end{array}$ & $\begin{array}{c}\text { Costos } \\
\text { relativos } \\
\text { unitarios } \\
\text { laborales } \\
\text { (OCDE) }\end{array}$ & SDRWPI* \\
\hline 1989 Q1 & 137.340437 & 124.033572 & 124.224636 & 167.616493 & 145.376735 \\
\hline 1989 Q2 & 135.105104 & 123.071874 & 125.977364 & 163.05234 & 142.908439 \\
\hline 1989 Q3 & 135.948257 & 121.980971 & 127.796589 & 158.805781 & 142.198011 \\
\hline 1989 Q4 & 138.0897 & 122.95082 & 128.789602 & 155.400155 & 142.504848 \\
\hline 1990 Q1 & 134.376766 & 122.865217 & 124.16294 & 159.821 & 133.287325 \\
\hline 1990 Q2 & 133.667361 & 120.274225 & 122.871271 & 158.202816 & 130.689837 \\
\hline 1990 Q3 & 136.346961 & 119.208456 & 122.340142 & 155.569384 & 133.45075 \\
\hline 1990 Q4 & 139.110849 & 118.802471 & 120.949244 & 153.186275 & 136.065075 \\
\hline 1991 Q1 & 130.856278 & 115.322519 & 114.596631 & 150.127608 & 124.328257 \\
\hline
\end{tabular}




\begin{tabular}{|c|c|c|c|c|c|}
\hline 1991 Q2 & 122.620368 & 109.701247 & 110.465611 & 140.350877 & 115.757181 \\
\hline 1991 Q3 & 121.310664 & 107.766363 & 109.7704 & 135.482997 & 113.59376 \\
\hline 1991 Q4 & 122.206219 & 106.337729 & 108.08954 & 132.749237 & 112.350894 \\
\hline 1992 Q1 & 117.202061 & 103.448276 & 103.146803 & 131.85654 & 107.814002 \\
\hline 1992 Q2 & 116.531561 & 102.165917 & 102.445875 & 127.437237 & 107.312453 \\
\hline 1992 Q3 & 119.580109 & 102.016527 & 102.870932 & 123.046635 & 109.4559 \\
\hline 1992 Q4 & 113.396778 & 98.0969198 & 100.100932 & 116.482236 & 102.948748 \\
\hline 1993 Q1 & 105.790152 & 95.6663159 & 96.4917528 & 117.439812 & 99.03358 \\
\hline 1993 Q2 & 99.933104 & 96.2124371 & 96.6253593 & 115.874855 & 99.6020446 \\
\hline 1993 Q3 & 97.7931106 & 93.6504963 & 95.0999738 & 112.879558 & 97.44945 \\
\hline 1993 Q4 & 96.1595703 & 91.6786358 & 94.1213676 & 110.840168 & 95.3811702 \\
\hline 1994 Q1 & 95.1716651 & 90.9614627 & 94.1213676 & 114.902907 & 96.117395 \\
\hline 1994 Q2 & 101.383348 & 98.2382605 & 99.0008831 & 118.20331 & 102.853775 \\
\hline 1994 Q3 & 105.149046 & 99.6578414 & 100.939566 & 117.688596 & 106.410777 \\
\hline 1994 Q4 & 110.952741 & 103.288001 & 106.622062 & 121.197431 & 112.703308 \\
\hline 1995 Q1 & 171.786228 & 160.333494 & 162.602974 & 191.38756 & 172.302608 \\
\hline 1995 Q2 & 158.243791 & 146.141855 & 144.405542 & 187.476565 & 157.551057 \\
\hline 1995 Q3 & 146.319496 & 134.547249 & 135.889155 & 180.018002 & 143.683016 \\
\hline 1995 Q4 & 160.482023 & 146.470071 & 149.851392 & 202.061022 & 156.378554 \\
\hline 1996 Q1 & 150.299062 & 139.899273 & 141.463905 & 195.3125 & 142.72512 \\
\hline 1996 Q2 & 139.653581 & 131.492439 & 132.404665 & 182.982617 & 131.319178 \\
\hline 1996 Q3 & 135.899523 & 126.305153 & 128.436726 & 173.882803 & 126.908534 \\
\hline 1996 Q4 & 133.874933 & 124.409057 & 127.249112 & 169.43409 & 125.09016 \\
\hline
\end{tabular}

Cuadro A2

(Continuación)

\begin{tabular}{|c|r|c|c|c|c|}
\hline Periodo & $\begin{array}{c}\text { Banco de } \\
\text { México }\end{array}$ & $\begin{array}{c}\text { Índices } \\
\text { relativos de } \\
\text { precios al } \\
\text { consumidor } \\
\text { (FMI) }\end{array}$ & $\begin{array}{c}\text { Índice } \\
\text { relativo de } \\
\text { precios al } \\
\text { consumidor } \\
\text { (OCDE) }\end{array}$ & $\begin{array}{c}\text { Costos } \\
\text { relativos } \\
\text { unitarios } \\
\text { laborales } \\
\text { (OCDE) }\end{array}$ & SDRWPI* \\
\hline 1997 Q1 & 122.0116 & 118.268548 & 118.940049 & 159.872102 & 116.243166 \\
\hline 1997 Q2 & 118.861961 & 115.5446 & 116.041657 & 153.468386 & 112.899032 \\
\hline 1997 Q3 & 112.83285 & 109.88206 & 111.194396 & 145.327714 & 107.456815 \\
\hline
\end{tabular}




\begin{tabular}{|c|c|c|c|c|c|}
\hline 1997 Q4 & 112.882037 & 109.377279 & 111.305797 & 145.327714 & 106.885827 \\
\hline 1998 Q1 & 109.904461 & 109.170306 & 109.234809 & 144.948543 & 101.604497 \\
\hline 1998 Q2 & 109.830998 & 108.932462 & 109.434047 & 143.184422 & 100.324057 \\
\hline 1998 Q3 & 116.324713 & 114.133536 & 116.014732 & 149.231458 & 105.255145 \\
\hline 1998 Q4 & 123.274246 & 117.30664 & 118.761136 & 151.14873 & 108.812367 \\
\hline 1999 Q1 & 114.390979 & 111.665302 & 111.005151 & 141.823855 & 101.926516 \\
\hline 1999 Q2 & 103.777474 & 102.95127 & 102.800428 & 127.975429 & 93.5434473 \\
\hline 1999 Q3 & 101.796772 & 100.020004 & 100.563993 & 122.159785 & 92.9705722 \\
\hline 1999 Q4 & 101.97495 & 99.950025 & 99.9642128 & 119.005117 & 93.7741797 \\
\hline 2000 Q1 & 97.065588 & 97.5039002 & 96.8124501 & 114.298777 & 90.542854 \\
\hline $2000 \mathrm{Q} 2$ & 95.6911623 & 97.4595543 & 97.1825798 & 112.410072 & 90.272506 \\
\hline 2000 Q3 & 91.4194639 & 93.8144975 & 93.856351 & 107.020548 & 87.2130171 \\
\hline $2000 \mathrm{Q} 4$ & 89.1210397 & 93.4433889 & 92.9980814 & 106.100796 & 86.0766415 \\
\hline $2001 \mathrm{Q} 1$ & 90.6099178 & 95.1927653 & 93.9592649 & 106.349038 & 85.2929926 \\
\hline $2001 \mathrm{Q} 2$ & 83.3981912 & 88.7784091 & 88.4467338 & 98.804466 & 78.2952862 \\
\hline $2001 \mathrm{Q} 3$ & 83.7871574 & 88.2223202 & 88.1401287 & 97.713504 & 77.1211036 \\
\hline 2001 Q4 & 82.5583902 & 87.4482598 & 86.5308651 & 95.6754688 & 74.5895202 \\
\hline 2002 Q1 & 77.839888 & 85.3218054 & 83.9825887 & 91.4076782 & 72.2580656 \\
\hline $2002 \mathrm{Q} 2$ & 79.9945559 & 88.1626895 & 87.7405407 & 93.7207123 & 76.0150867 \\
\hline 2002 Q3 & 85.8587149 & 92.075379 & 91.573079 & 96.9649956 & 80.8420529 \\
\hline $2002 \mathrm{Q} 4$ & 87.2747638 & 94.1649142 & 92.719309 & 98.4930562 & 82.2797537 \\
\hline 2003 Q1 & 95.4303267 & 100.887813 & 99.4142512 & 105.385183 & 90.8185125 \\
\hline 2003 Q2 & 94.3926004 & 97.2258232 & 96.8499552 & 101.832994 & 88.2179441 \\
\hline 2003 Q3 & 96.3120679 & 99.1735537 & 99.0761152 & 102.976007 & 90.3136292 \\
\hline 2003 Q4 & 102.970691 & 104.155817 & 103.221331 & 107.192625 & 96.510013 \\
\hline 2004 Q1 & 102.313461 & 102.235551 & 101.174453 & 104.123282 & 97.7135354 \\
\hline 2004 Q2 & 104.771539 & 105.23731 & 104.914323 & 105.152471 & 101.679823 \\
\hline 2004 Q3 & 105.382752 & 105.229927 & 105.278806 & 104.755919 & 102.525226 \\
\hline 2004 Q4 & 105.898762 & 104.657248 & 103.799907 & 103.87452 & 102.435652 \\
\hline
\end{tabular}




\section{Cuadro A2}

(Continuación)

\begin{tabular}{|c|c|c|c|c|c|}
\hline Periodo & $\begin{array}{c}\text { Banco de } \\
\text { México }\end{array}$ & $\begin{array}{l}\text { Índices } \\
\text { relativos de } \\
\text { precios al } \\
\text { consumidor } \\
\text { (FMI) }\end{array}$ & $\begin{array}{l}\text { Índice } \\
\text { relativo de } \\
\text { precios al } \\
\text { consumidor } \\
(O C D E)\end{array}$ & $\begin{array}{c}\text { Costos } \\
\text { relativos } \\
\text { unitarios } \\
\text { laborales } \\
(\text { OCDE) }\end{array}$ & $S D R W P I^{*}$ \\
\hline 2005 Q1 & 105.251225 & 103.684247 & 102.635137 & 102.679947 & 102.983869 \\
\hline 2005 Q2 & 101.739025 & 100.600248 & 100.973547 & 100.775975 & 100.663127 \\
\hline 2005 Q3 & 97.7444672 & 98.0552378 & 98.8410882 & 98.5124618 & 98.0173636 \\
\hline 2005 Q4 & 95.2652833 & 97.8792822 & 97.6952709 & 98.1643271 & 98.33564 \\
\hline 2006 Q1 & 93.9717488 & 96.6712854 & 96.2935644 & 97.2951936 & 97.3751085 \\
\hline $2006 \mathrm{Q} 2$ & 102.32338 & 102.96187 & 103.796319 & 102.701037 & 107.366918 \\
\hline 2006 Q3 & 100.363331 & 100.623868 & 101.413872 & 99.631364 & 106.273669 \\
\hline 2006 Q4 & 98.3937255 & 99.1047537 & 98.7988041 & 98.1161695 & 103.735322 \\
\hline 2007 Q1 & 99.352191 & 100.324382 & 99.7249586 & 98.5804416 & 105.17037 \\
\hline 2007 Q2 & 100.869648 & 100.277434 & 100.864906 & 97.8760889 & 108.722401 \\
\hline 2007 Q3 & 102.550066 & 101.033914 & 101.844215 & 99.7108386 & 109.196028 \\
\hline 2007 Q4 & 103.722535 & 101.902174 & 101.270089 & 100.704935 & 109.712153 \\
\hline 2008 Q1 & 105.360514 & 102.487018 & 101.383027 & 102.322726 & 112.880592 \\
\hline $2008 \mathrm{Q} 2$ & 103.966613 & 99.2260369 & 99.5594494 & 100.735368 & 114.471472 \\
\hline $2008 \mathrm{Q} 3$ & 100.022782 & 96.3855422 & 97.2960456 & 100.010001 & 110.92322 \\
\hline 2008 Q4 & 115.418062 & 113.726828 & 113.844298 & 123.167878 & 117.130404 \\
\hline 2009 Q1 & 123.96173 & 123.558484 & 122.469984 & 134.661998 & 120.82549 \\
\hline 2009 Q2 & 117.434363 & 114.889706 & 115.089009 & 124.750499 & 114.617642 \\
\hline 2009 Q3 & 120.138783 & 115.247205 & 116.17647 & 124.533001 & 117.934728 \\
\hline 2009 Q4 & 119.933022 & 115.269346 & 114.745641 & 123.00123 & 118.695204 \\
\hline 2010 Q1 & 112.810249 & 110.444354 & 109.525942 & 118.722545 & 113.859879 \\
\hline 2010 Q2 & 107.98907 & 106.224772 & 107.285524 & 115.180834 & 110.875914 \\
\hline 2010 Q3 & 112.29433 & 108.506944 & 109.842745 & 117.577895 & 115.052762 \\
\hline 2010 Q4 & 110.371828 & 106.330191 & 106.432923 & 115.167569 & 114.230199 \\
\hline 2011 Q1 & 107.584025 & 104.369608 & 103.810683 & 112.574581 & 114.761328 \\
\hline $2011 \mathrm{Q} 2$ & 108.380754 & 102.799575 & 103.717376 & 110.314396 & 115.598425 \\
\hline 2011 Q3 & 113.274807 & 106.795771 & 108.830626 & 115.955473 & 119.970742 \\
\hline
\end{tabular}

Fuente: Banco de México, IMF y OCDE.

* Estimación propia con datos del IMF. 